OPEN ACCESS

Edited by: Giovanni Battista Luciani, University of Verona, Italy

Reviewed by:

Benjamin Bierbach, University Hospital Bonn, Germany Benjamim Ficial,

Integrated University Hospital Verona, Italy

*Correspondence: Vidya R. Raghavan vidya.raghavan@childrens.harvard.edu

Specialty section: This article was submitted to

Pediatric Cardiology, a section of the journal

Frontiers in Pediatrics

Received: 17 February 2021 Accepted: 21 June 2021 Published: 26 July 2021

Citation:

Raghavan VR, da Cruz EM, Kaufman J and Osorio Lujan S (2021) International Survey on the Use of

Arginine Vasopressin in the Postoperative Management of Single Ventricle Patients.

Front. Pediatr. 9:669055.

doi: 10.3389/fped.2021.669055

\section{International Survey on the Use of Arginine Vasopressin in the Postoperative Management of Single Ventricle Patients}

\author{
Vidya R. Raghavan ${ }^{1 *}$, Eduardo M. da Cruz ${ }^{1,2}$, Jon Kaufman ${ }^{1,2}$ and Suzanne Osorio Lujan ${ }^{1,2}$ \\ ${ }^{1}$ Department of Pediatrics, University of Colorado Denver School of Medicine, Aurora, CO, United States, ${ }^{2}$ The Heart \\ Institute, Children's Hospital Colorado, Aurora, CO, United States
}

Management of patients with single ventricle physiology after surgical palliation is challenging. Arginine vasopressin has gained popularity in recent years as a non-catecholamine vasoactive medication due to its unique properties. However, data regarding its use in the pediatric population is limited. Therefore, we designed a survey to explore whether and how clinicians use this medication in intensive care units for the postoperative management of single ventricle patients. This international survey aimed to assess usage, practices, and concepts related to arginine vasopressin in pediatric intensive care units worldwide. Directors of pediatric intensive care units who are members of the following international professional societies: European Society of Pediatric Neonatal Intensive Care, Association for European Pediatric and Congenital Cardiology, and Pediatric Cardiac Intensive Care Society were invited to participate in this survey. Of the 62 intensive care unit directors who responded, nearly half use arginine vasopressin in the postoperative management of neonatal single ventricle patients, and 90\% also use the drug in subsequent surgical palliation. The primary indications are vasoplegia, hemodynamic instability, and refractory shock, although it is still considered a second-line medication. Conceptual benefits include improved hemodynamics and end-organ perfusion and decreased incidence of low cardiac output syndrome. Those practitioners who do not use arginine vasopressin cite lack of availability, fear of potential adverse effects, unclear indication for use, and lack of evidence suggesting improved outcomes. Both users and non-users described increased myocardial afterload and extreme vasoconstriction as potential disadvantages of the medication. Despite the lack of conclusive data demonstrating enhanced clinical outcomes, our study found arginine vasopressin is used widely in the care of infants and children with single ventricle physiology after the first stage and subsequent palliative surgeries. While many intensive care units use this medication, few had protocols, offering an area for further growth and development.

Keywords: arginine vasopressin, single ventricle, survey, international, pediatric 


\section{INTRODUCTION}

Infants and children with single ventricle physiology are among the most challenging for providers who care for patients with congenital heart disease. Although these malformations are relatively rare, affecting only 5 of every 100,000 children, their management is complex with patients usually requiring surgical palliation in the neonatal period $(1,2)$. In the immediate aftermath of surgery, most of these patients suffer from shock states, myocardial depression, and hemodynamic instability. The mainstays of postoperative management include supporting the myocardium and peripheral circulation with vasoactive and inotropic agents (1). Arginine vasopressin (AVP) is a non-catecholamine vasoactive whose use has gained popularity in the last decade due to its unique properties and safety profile. Yet, data is scarce, particularly concerning the single ventricle population.

Compared to other vasoactive agents, AVP offers the unique effects of a vasoconstrictor of venous capacitance that increases systemic vascular resistance without a direct impact on systolic function; nonetheless, it may alter heart rate or myocardial oxygen consumption in some circumstances. Indeed, AVP, a neurohypophyseal hormone secreted by the pituitary gland, acts on V1 receptors and causes smooth muscle-mediated vasoconstriction (3). This is achieved via a mechanism of inhibition of inducible and endogenous nitric oxide synthetase at the cellular level (4). The efficacy with which it causes selective vasoconstriction has led some to recommend AVP for hypoxemia in postoperative Norwood patients as a means of increasing pulmonary blood flow by raising systemic vascular resistance, thus increasing the $\mathrm{Qp} / \mathrm{Qs}$ (5). As systemic vascular resistance increases under the effect of AVP, caution ought to be exerted with regards to the subsequent increase of the systemic ventricle afterload that may ultimately negatively impact systolic function and stroke volume, notably in the setting of suboptimal preload and systemic ventricle dysfunction. Owing to these effects, AVP is of interest in patients with significant postoperative systemic inflammatory disorders and vasoplegia in whom modulation of the vascular tone is sought. A more recent retrospective study exploring the use of AVP in Norwood patients reported that the initiation of AVP in their postoperative course was temporally associated with improvement in markers of perfusion, including systolic blood pressure, urine output, lactic acid, and $\mathrm{pH}$ (6).

Albeit AVP is commonly used in the cardiovascular setting, evidence-based data remains scarce. There is no formal data about its use internationally or the protocols and practices accepted and applied in the cardiac care in intensive care units. The objective of this study was to explore the prevalence, concepts, and methods of AVP use in the postoperative management of pediatric single ventricle patients in intensive care units worldwide.

\section{MATERIALS AND METHODS}

\section{Study Design}

We performed a cross-sectional international survey aimed at neonatal, pediatric, and cardiac intensive care unit directors regarding their use of AVP. The survey inquired about attitudes regarding safety, indications for use, benefits, advantages, and disadvantages. This study was deemed exempt by the University of Colorado Institutional Review Board.

\section{Survey Development}

We designed an original survey to assess use and concepts regarding AVP in the pediatric intensive care unit after cardiac surgery. The questions were developed by the authors based on their experience in designing international surveys currently published in peer-reviewed journals and their own experience with the use of AVP in the single ventricle patient population in a large university-affiliated free-standing pediatric hospital. The survey was built with a dynamic branching logic format to provide follow-up questions specific to the survey respondents' usage practices (Supplementary Figure 1). The survey was also tested for length and took 7-10 min to complete. All survey questions required a response before proceeding with the subsequent question to ensure comprehensive data gathering.

\section{Study Subjects}

Participants were recruited through the following international societies: European Society of Pediatric Neonatal Intensive Care (ESPNIC), Association for European Pediatric and Congenital Cardiology (AEPC), and Pediatric Cardiac Intensive Care Society (PCICS) directories. The authors utilized a publicly available list to distribute the survey for PCICS; it was distributed with endorsement by AEPC and ESPNIC. The respondents reflect a convenience sample. The survey was provided through a link to Survey Monkey ${ }^{\mathrm{TM}}$ conducted between November 2017 and January 2018. Survey responses were collected anonymously, except for the respondents' country of practice.

\section{Statistical Analysis}

Responses for each completed survey were collected and managed using REDCap electronic data capture tools hosted at the University of Colorado REDCap (Research Electronic Data Capture). It is a secure, web-based application designed to support data capture for research studies, providing: (1) an intuitive interface for validated data entry; (2) audit trails for tracking data manipulation and export procedures; (3) automated export procedures for seamless data downloads to standard statistical packages, and (4) procedures for importing data from external sources (7). A descriptive statistical analysis (distribution of responses expressed as percentages) was performed with PRISM and Microsoft Excel (2019). Both fully and partially completed surveys were analyzed and none were excluded. The authors report the percentage (\%) for all responses.

\section{RESULTS}

There were 62 survey respondents from 21 different countries spanning five continents (Table 1). Almost half of the respondents (48.4\%) use AVP for the postoperative care of neonatal patients undergoing a Norwood operation with a Sano or Blalock-Taussig shunt placement. Among AVP users, the majority (90\%) also used AVP for subsequent interventions such 
TABLE 1 | Geographic distribution of survey respondents by country and region.

\begin{tabular}{|c|c|}
\hline Region and Country & Number of survey participants, $n(\%)$ \\
\hline Europe & $29(46.8 \%)$ \\
\hline United Kingdom & 7 \\
\hline Switzerland & 4 \\
\hline France & 3 \\
\hline Italy & 3 \\
\hline Netherlands & 3 \\
\hline Spain & 3 \\
\hline Germany & 2 \\
\hline Belgium & 1 \\
\hline Greece & 1 \\
\hline Latvia & 1 \\
\hline Portugal & 1 \\
\hline North America & 25 (40.3\%) \\
\hline United States & 23 \\
\hline Canada & 2 \\
\hline Central and South America & $4(6.5 \%)$ \\
\hline Argentina & 1 \\
\hline Colombia & 1 \\
\hline Costa Rica & 1 \\
\hline Mexico & 1 \\
\hline Asia & $3(4.8 \%)$ \\
\hline Saudi Arabia & 1 \\
\hline Singapore & 1 \\
\hline United Arab Emirates & 1 \\
\hline Australia & $1(1.6 \%)$ \\
\hline Australia & 1 \\
\hline Total respondents & 62 \\
\hline
\end{tabular}

as partial or total cavo-pulmonary connections. Interestingly, a small proportion (9.4\%) of those who did not use AVP for the neonatal intervention did use it for management in subsequent palliations (Figure 1). Within the group of AVP users, only $27.2 \%$ reported standardized practice at their institution, and among them, only one had an institutional protocol for AVP use in these patients.

The most frequent indications for AVP use included evidence of vasoplegia or excessive systemic vasodilation (90.6\%), refractory shock (68.8\%), and hemodynamic instability (75.0\%). Respondents also provided the following write-in responses, including "optimize renal perfusion," "routine post-op management post-Fontan," and "reduce catecholamine doses if tachyarrhythmias present” (Table 2).

The majority of AVP users reported 0.0003 to 0.0006 $\mathrm{U} / \mathrm{kg} / \mathrm{min}$ of AVP as the most commonly used dosage (54.8\%). A minority of respondents used $<0.0003 \mathrm{U} / \mathrm{kg} / \mathrm{min}(16.4 \%)$, 0.0006 to $0.0009 \mathrm{U} / \mathrm{kg} / \mathrm{min}$ (12.9\%), or $>0.0009 \mathrm{U} / \mathrm{kg} / \mathrm{min}(9.7 \%)$ (Table 2).

There was a consensus among users that AVP was regarded as a second-line drug by $90 \%$. Similarly, the presence of an umbilical artery catheter or umbilical vein catheter was not considered a contraindication to AVP use by $93.5 \%$. An echocardiogram was also not routinely obtained before starting AVP according to the majority of respondents, $84.4 \%$. Enteral feeding while receiving AVP was divisive, with 58.1\% reporting that they feed, while the remaining $41.9 \%$ consider enteral nutrition to be a contraindication (Table 2).

Amongst AVP non-users, the most common reasons for not using the drug included lack of availability, concern about potential adverse effects, absence of clear indication for use, and absence of evidence suggesting improved outcomes (Table 3). Alternate first-line drugs used instead of vasopressin included norepinephrine and phenylephrine (Table 3).

AVP users reported several perceived benefits including improved hemodynamic profile, counteraction of the potential vasodilatory effects of milrinone, improved end-organ perfusion, decreased incidence of low cardiac output syndrome, and impact on pulmonary hypertension in postoperative patients (Figure 2).

The most common described disadvantages cited by both AVP users and non-users included extreme vasoconstriction and increased myocardial afterload: 61.3 and 57.1\%, respectively. In a multiple-choice option question, AVP users also reported sodium abnormalities (45.2\%), increased risk of necrotizing enterocolitis $(29.0 \%)$, oliguria $(25.8 \%)$, increased risk of low cardiac output syndrome $(22.6 \%)$, and coronary or splanchnic ischemia $(22.6 \%)$ as other disadvantages. AVP non-users also perceived increased risk of necrotizing enterocolitis, coronary or splanchnic ischemia, and oliguria as disadvantages and reasons not to use this medication (Figure 3).

\section{DISCUSSION}

This study attempts to understand the concepts around the use of the AVP in a population of single ventricle patients for which there is controversy about the virtues or the disadvantages of the drug. This manuscript shares descriptive data about these concepts and attempts to better characterize practices to promote consistency in the future. It provides a valuable insight into practices with AVP in 63 programs across 21 different countries.

In the high-risk and challenging population of postoperative palliation of single ventricle patients, AVP seems to be of interest among pediatric cardiac intensivists for its unique properties. Vasopressin has a wide range of dose-dependent physiologic effects on the cardiovascular system, arguably by increasing vascular tone and improving inotropy. Moreover, it does so without directly increasing myocardial oxygen demand or heart rate, although changes in those parameters may be indirectly induced $(3,5,6,8-14)$. The virtues of the drug are not merely restricted to the effects on the vasculature. V2 and V3 receptors in the renal collecting duct are stimulated by AVP to reabsorb urea, sodium, and water $(14,15)$. In addition, stimulation of cardiac oxytocin receptors, which have been localized in all cardiac chambers, leads to the production of atrial natriuretic peptide (10). Last but not least, purinergic receptors also bind AVP (8). Indeed, AVP binds to $\mathrm{V}_{1}$ receptors on vascular smooth muscle to cause vasoconstriction through the $\mathrm{IP}_{3}$ signal transduction pathway and Rho-kinase pathway, which increases arterial pressure. Purinergic receptors are also present in the heart and coronary arteries, although there are conflicting data 


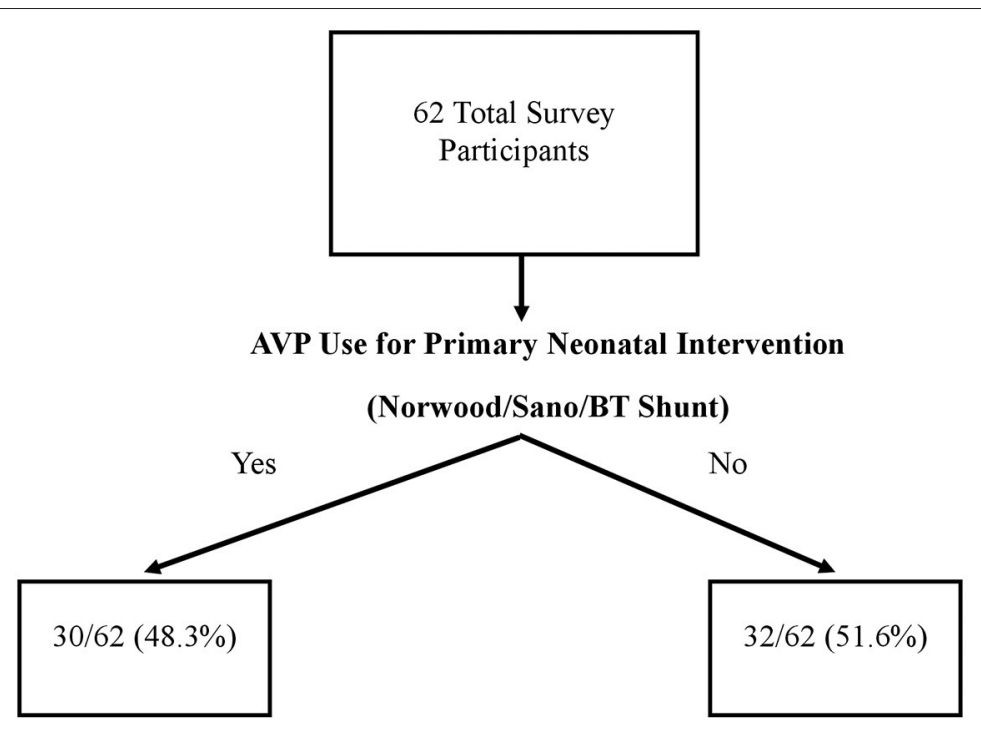

AVP Use for Subsequent Intervention

(Partial and Total Cavo-pulmonary Connection)
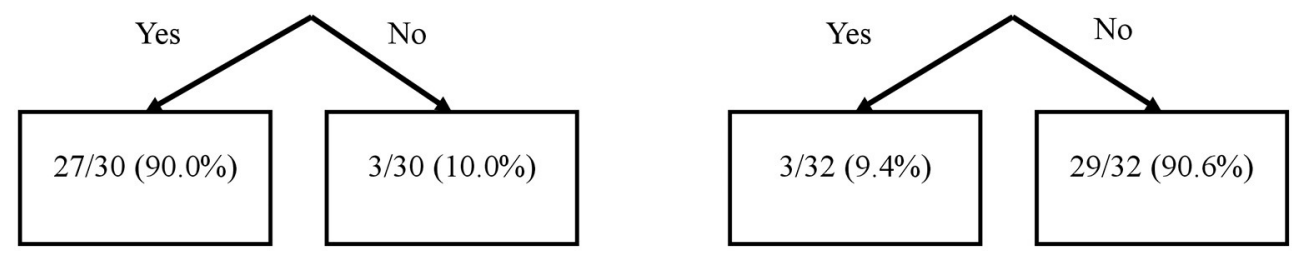

FIGURE 1 | Prevalence of Arginine Vasopressin (AVP) use among survey respondents.

regarding the activation of these receptors and the subsequent clinical effect $(12,13,16)$.

Some reports have shown an absolute or relative deficiency of vasopressin after cardiopulmonary bypass surgery, and the use of exogenous AVP resulted in improvement in the hemodynamic status. These data support AVP use for neonates with vasodilatory or catecholamine-resistant shock after cardiopulmonary bypass $(5,17,18)$. This would seem to agree with Alten et al. and with Grant et al., whose studies on the routine use of AVP in the postoperative period reported lower use of fluid resuscitation and catecholamines $(6,19)$. However, Morrison et al. seemed to contradict these findings by demonstrating that vasopressin levels in pediatric patients after cardiopulmonary bypass are markedly elevated. Additionally, their results suggested that exogenous use of AVP was ineffective or potentially detrimental in its effects on cardiac output and organ perfusion (20). As reflected in our survey, each of these studies note that there is little consensus regarding appropriate dosing, indications for use, risks or benefits, and this lack of consistency has been well-documented (21).

Rosenzweig et al., and Lechner et al., have previously characterized the effect of vasopressin on excessive vasodilation and refractory shock in a population of neonates with catecholamine-resistant vasodilation after cardiopulmonary bypass. In addition, both described improved blood pressure in the patient population that received AVP, which supports this indication $(17,18)$. Thus, the findings of these studies echo the indications for AVP use cited by survey respondents.

To the best of our knowledge, no studies have determined the appropriate dosage of AVP in pediatric patients. Among the pediatric studies mentioned, dosages have ranged between 0.0003 and $0.002 \mathrm{U} / \mathrm{kg} / \mathrm{min}$, primarily extrapolated from adult literature. Lechner et al. reported no ischemic effects with dosages up to $0.001 \mathrm{U} / \mathrm{kg} / \mathrm{min}$ (17). Our survey determined ranges based on our experiences with AVP in the cardiac intensive care unit at Children's Hospital Colorado. The survey results show that AVP users reported low-dose AVP (0.0003-0.0006 U/kg/min) as the most frequently used dose. Dosages of $<0.0003 \mathrm{U} / \mathrm{kg} / \mathrm{min}$ and $>0.0009 \mathrm{U} / \mathrm{kg} / \mathrm{min}$ were not as commonly reported. We believe this is the first study to describe the most widely used AVP dosage for pediatric single ventricle patients after cardiopulmonary bypass with an international perspective.

The use of umbilical access via arterial or venous catheter was not considered a contraindication to the majority of AVP users. Most providers did not obtain an echocardiogram before the initiation of AVP to document the presence or absence of 
TABLE 2 | Arginine Vasopressin (AVP) Practice Patterns and Indications for Use Among Users.

\begin{tabular}{|c|c|}
\hline AVP practice patterns and indications & $\%$ Among users \\
\hline \multicolumn{2}{|l|}{$\begin{array}{l}\text { Consistent practice amongst your colleagues } \\
\text { regarding AVP usage }\end{array}$} \\
\hline Consistent Practice for AVP Usage & $27.3 \%$ \\
\hline No Consistent Practice & $72.7 \%$ \\
\hline \multicolumn{2}{|l|}{$\begin{array}{l}\text { Frequency of echocardiography prior to AVP } \\
\text { administration }\end{array}$} \\
\hline Echo routinely obtained & $15.6 \%$ \\
\hline Echo not routinely obtained & $84.4 \%$ \\
\hline \multicolumn{2}{|l|}{ Indications for AVP among users* } \\
\hline Evidence of vasoplegia & $93.5 \%$ \\
\hline Postoperative hemodynamic instability & $77.4 \%$ \\
\hline Refractory Shock & $70.9 \%$ \\
\hline Routine postoperative management & $6.4 \%$ \\
\hline Routine intraoperative management & $3.2 \%$ \\
\hline \multicolumn{2}{|l|}{ AVP utilized as a first or second-line choice } \\
\hline First-line & $10.0 \%$ \\
\hline Second-line & $90.0 \%$ \\
\hline \multicolumn{2}{|l|}{ AVP dosage } \\
\hline Less than $0.0003 \mathrm{U} / \mathrm{kg} / \mathrm{min}$ & $12.9 \%$ \\
\hline $0.0003-0.0006 \mathrm{U} / \mathrm{kg} / \mathrm{min}$ & $54.8 \%$ \\
\hline $0.0006-0.0009 \mathrm{U} / \mathrm{kg} / \mathrm{min}$ & $16.2 \%$ \\
\hline Greater than $0.0009 \mathrm{U} / \mathrm{kg} / \mathrm{min}$ & $9.7 \%$ \\
\hline Other & $6.4 \%$ \\
\hline \multicolumn{2}{|l|}{ Relationship between AVP and enteral feeding } \\
\hline AVP is a contraindication to enteral feeding & $41.9 \%$ \\
\hline AVP is not a contraindication to enteral feeding & $58.1 \%$ \\
\hline \multicolumn{2}{|l|}{$\begin{array}{l}\text { Presence of umbilical arterial or venous catheters } \\
\text { contraindication to AVP usage }\end{array}$} \\
\hline Umbilical arterial catheters are a contraindication to AVP & $6.5 \%$ \\
\hline Neither are a contraindication for starting AVP & $93.5 \%$ \\
\hline
\end{tabular}

(*) More than one response accepted.

ventricular dysfunction or atrioventricular or semi-lunar valve regurgitation. This practice could be supported by the results of a retrospective study by Iliopoulos et al., which found that ventricular dysfunction was not a predictor of hemodynamic response to AVP (11). Initiation of enteral feeding while receiving vasopressin was fairly split between respondents. Reasons for avoiding enteral feeding may relate to the severity of the patient's baseline clinical condition rather than to the use of AVP, especially as most AVP users felt AVP was a second-line medication. Porcine models have shown a decrease in blood flow in the gastric mucosa after cardiopulmonary bypass, exacerbated by the use of $\operatorname{AVP}(9,22)$, which could pose a theoretical risk of necrotizing enterocolitis. However, Alten et al. reported no cases of necrotizing enterocolitis and adequate tolerance of enteral feeds in all patients with early initiation of AVP after cardiovascular bypass surgery (19).

AVP non-users reported the preferential use of catecholamines including epinephrine, norepinephrine, alpha1 -adrenergic agonist, and phenylephrine instead of AVP in the setting of a need for the vasoconstrictive effect of vascular beds. These drugs have similar sympathomimetic effects targeting
TABLE 3 | Reasons for Arginine Vasopressin (AVP) Avoidance and Alternate Medications Used Among Non-Users.

AVP avoidance and alternate medications

$\%$ Among non-users

Alternative agents to AVP

Norepinephrine $\quad 85.2 \%$

Phenylephrine $\quad 14.8 \%$

Primary reason AVP not used among non-users*

AVP not available

Concern about potential adverse events

Absence of clear indication for use

Absence of evidence suggesting improved

outcomes compared to other agents

Other

No response

\section{$14.8 \%$}

$27.2 \%$

$25.9 \%$

$22.2 \%$

$14.8 \%$

$7.4 \%$

$2.5 \%$

(*) More than one response accepted.

the peripheral vasculature as potent vasoconstrictors. There is also a long history and likely greater comfort with using these agents. Future studies should investigate the possibility of an age or experience bias or association with the use of AVP and the providers who administer it.

Drugs of this nature are an attractive alternative because the challenge of managing these single ventricle patients can be related to the clinical condition of vasoplegia and systemic inflammatory response syndrome as a result of cardiopulmonary bypass (23). Vasoplegia is a form of vasodilatory shock characterized by low systemic vascular resistance with preserved or increased cardiac output (24). The pathophysiology of postoperative vasoplegia in pediatric cardiac patients may be complex and is incompletely understood. It may occur secondary to a profound systemic inflammatory response induced by surgical trauma, exposure of blood to foreign surfaces of the cardiopulmonary bypass circuit, reperfusion injury, and possibly release of endotoxin from bacterial translocation. It may also reflect an absolute or relative deficiency of endogenous vasopressin in children $(25,26)$. These patients may develop catecholamine-resistant hypotension or extreme vasodilation, and several drugs may be required to treat it adequately but could ultimately be ineffective in managing the hemodynamic state.

The lack of availability of AVP in the pharmaceutical paraphernalia was the most common reason the drug was not utilized by non-users. Yet, in four of the five continents included, there was reported use of AVP, which suggests against specific geographic limitations to availability. The remaining responses reflect the need for further studies regarding the use of vasopressin in the pediatric population, including concern for potential adverse effects, absence of clear indication for use, and absence of evidence suggesting improved outcomes.

The benefits reported by AVP users included improved hemodynamics, counteracting vasoplegia, and improving endorgan perfusion, which echoes the findings of a previous publication from our group (6). However, it is worth noting that AVP non-users also reported these same benefits as AVP users, indicating that other factors influence their preference not to use the drug in the clinical setting. 


\section{Perceived Benefits of Arginine Vasopressin}

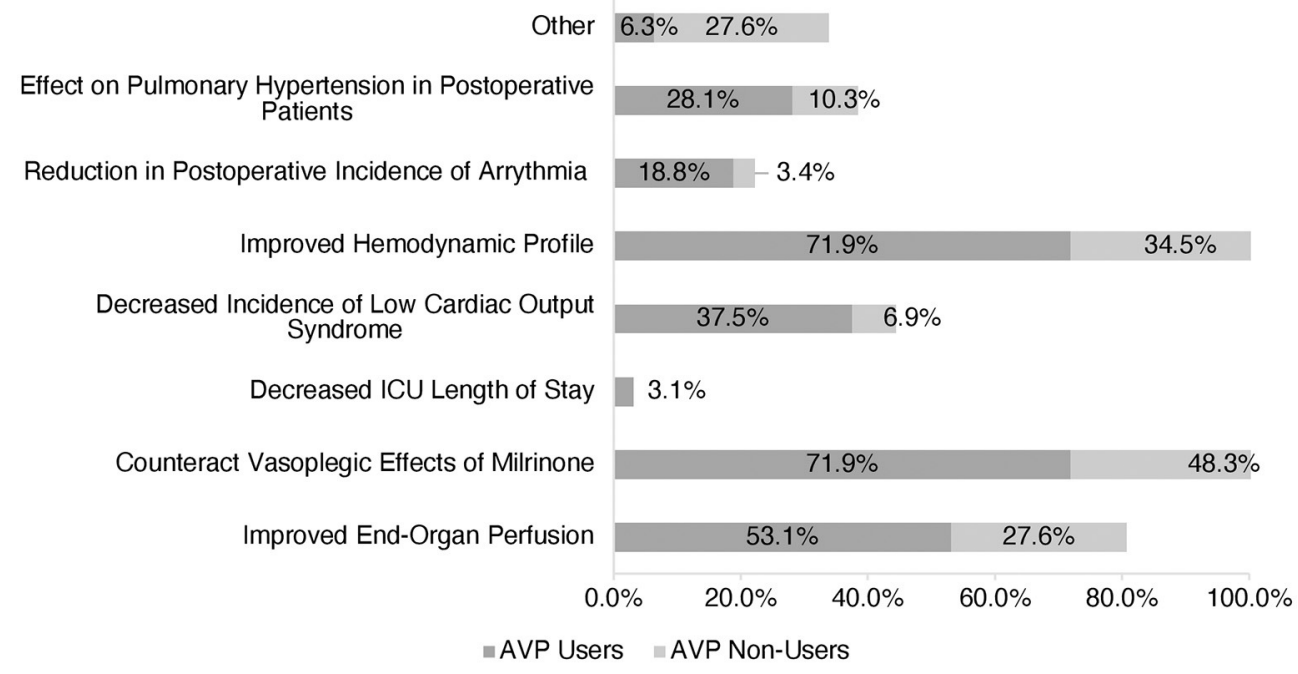

FIGURE 2 | Perception of Arginine Vasopressin (AVP) benefits amongst users vs. non-users. $\left(^{\star}\right)$ More than one response accepted.

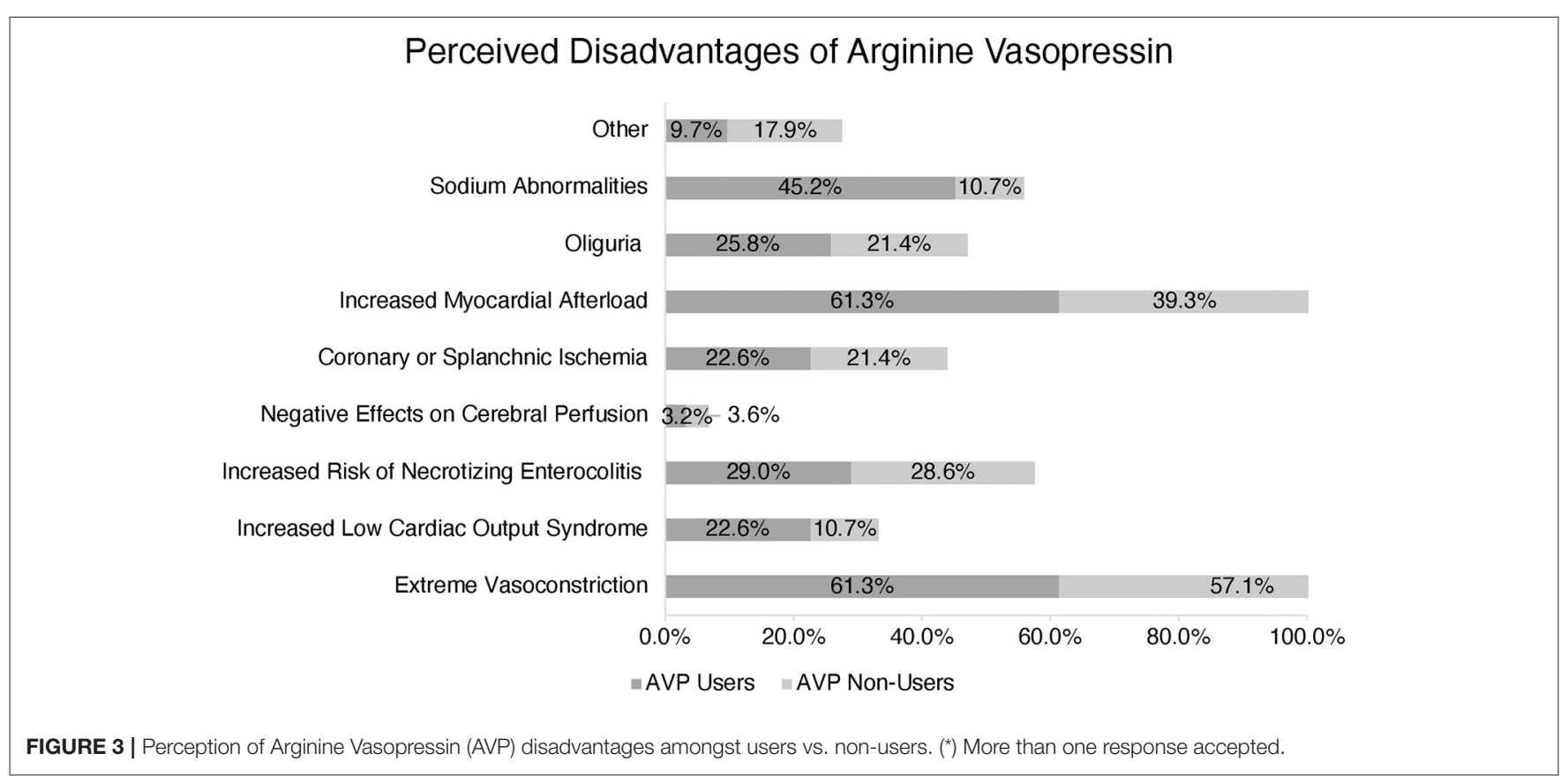

AVP users and non-users reported extreme vasoconstriction and increased myocardial afterload as potential adverse effects. Indeed, AVP must be used cautiously in the postoperative pediatric patient with evidence of ventricular dysfunction, and the potential for organ or extremity-specific deleterious vasoconstriction needs to be considered. However, it is worth noting that none of the studies in pediatric patients receiving AVP after cardiopulmonary bypass described such adverse effects in their subjects $(5,6,17-19)$. While AVP non-users selected necrotizing enterocolitis, oliguria, and splanchnic/coronary ischemia as the most common perceived disadvantages, these were less popular responses among AVP users.

AVP users also reported sodium abnormalities as a disadvantage, but this was not a frequent response from AVP non-users. Two retrospective studies have reported an increased incidence of hyponatremia associated with the patients who received vasopressin post-operatively $(6,27)$. Further studies should evaluate the effects of AVP on sodium balance in the pediatric cardiac intensive care unit.

Limitations of this study were inherent to the survey nature, which relies on the solicited subjects' willingness to respond 
and complete the survey and provide accurate responses. The methodology used also reflects a convenience sampling which may lend an inherent bias to the survey respondents. Also, answers may not have reflected the variations in practice within the Directors' programs. Although the survey was sent to the very large general membership of the societies, it is unknown how many Unit Directors were reached, making it impossible to calculate an accurate response rate, in spite of this, 62 responses from 21 countries seems remarkable to the authors. By targeting Unit Directors through the databases of the significant international societies related to the field, the study did not capture those not enlisted in such scholar societies. Although not capturing all practices around the world, this survey provides a unique background of consistencies and discrepancies in the use of AVP, which remains of great importance. A few of the survey respondents did not complete the entirety of the survey; thus, only the information entered could be collected and analyzed. Additionally, it may have been more illustrative to collect further information about the centers where the respondents practice, such as the number of surgeries performed and beds in the intensive care unit, to better contextualize their AVP use. It was not the nature of the study to analyze outcomes of stage 1 single ventricle palliation or correlation of the latter with the use of vasopressin. Last but not least, this survey depicts practices but also individual concepts rather than documented data. Notwithstanding that fact, it does provide for the first time in literature documentation of international practices regarding AVP among single ventricle pediatric patients.

\section{CONCLUSIONS}

Even in the absence of robust investigational data to support the routine use of AVP in pediatric patients after cardiac surgery, this survey responses demonstrate that AVP use is relatively widespread in the postoperative management of patients with single ventricle congenital heart disease. It also suggests higher general interest and ubiquity of use than was previously described. Major highlights of this study relate to the fact that approximately half of the surveyed practitioners use AVP to manage the first-stage palliation for single ventricle patients, albeit many consider AVP as a second-line drug. Notwithstanding that fact, the vast majority (90\%) of these clinical providers also use AVP in subsequent interventions, which may reflect a higher level of trust in the drug in patients beyond the neonatal period. Of significant importance, we documented that protocols are scarcely used in relation to AVP, which opens avenues toward implementing more consistent practices within the medical community in charge of the postoperative course of single ventricle

\section{REFERENCES}

1. Walker SG, Stuth EA. Single-ventricle physiology: perioperative implications. Semin Pediatr Surg. (2004) 13:188-202. doi: 10.1053/j.sempedsurg.2004. 04.005

2. Marelli AJ, Mackie AS, Ionescu-Ittu R, Rahme E, Pilote L. Congenital heart disease in the general population: changing prevalence and age distribution. patients. It is clear that most indications to use AVP in the postoperative period of single ventricle interventions relate to the optimization of tissue perfusion and end-organ function and to antagonize excessive systemic vasodilation secondary to the postoperative inflammatory status allied with side-effects of other cardiovascular drugs. By no means does this survey provide evidence-based data, nor should it influence clinical practices. However, it may spawn clinical studies designed to explore the impacts of AVP on patient outcomes. Nonetheless, a study of this nature may foster further collaboration to implement consistent algorithms for AVP use, gather data collectively, and hopefully better understand the characteristics of this drug and its relation with outcomes in this very complex patient population.

\section{DATA AVAILABILITY STATEMENT}

The raw data supporting the conclusions of this article will be made available by the authors, without undue reservation.

\section{ETHICS STATEMENT}

The studies involving human participants were reviewed and approved by University of Colorado School of Medicine Institutional Review Board. The patients/participants provided their written informed consent to participate in this study.

\section{AUTHOR CONTRIBUTIONS}

VR conceptualized the study and designed the survey along with EC and SO. VR designed the data collection instrument, collected the data, carried out the initial analyses, drafted the initial manuscript, and reviewed and revised the manuscript. SO coordinated and supervised data collection and reviewed and revised the manuscript. JK and EC critically reviewed the survey design and manuscript for intellectual content. All authors approved the final manuscript as submitted and agreed to be accountable for all aspects of the work.

\section{FUNDING}

This project was supported by NIH/NCRR Colorado CTSI Grant Number UL1 RR025780.

\section{SUPPLEMENTARY MATERIAL}

The Supplementary Material for this article can be found online at: https://www.frontiersin.org/articles/10.3389/fped. 2021.669055/full\#supplementary-material
Circulation. (2007) 115:163-72. doi: 10.1161/CIRCULATIONAHA.106.6 27224

3. Thibonnier M. Signal transduction of V1-vascular vasopressin receptors. Regul Pept. (1992) 38:1-11. doi: 10.1016/0167-0115(92)90067-5

4. Koshimizu TA, Nakamura K, Egashira N, Hiroyama M, Nonoguchi H, Tanoue A. Vasopressin V1a and V1b receptors: from molecules to physiological systems. Physiol Rev. (2012) 92:1813-64. doi: 10.1152/physrev.00035.2011 
5. Mastropietro CW, Clark JA, Delius RE, Walters HL III, Sarnaik AP. Arginine vasopressin to manage hypoxemic infants after stage I palliation of single ventricle lesions. Pediatr Crit Care Med. (2008) 9:506-10. doi: 10.1097/PCC.0b013e3181849ce0

6. Burton GL, Kaufman J, Goot BH, da Cruz. E.M. The use of arginine vasopressin in neonates following the Norwood procedure. Cardiol Young. (2011) 21:536-44. doi: 10.1017/S1047951111000370

7. Harris PA, Taylor R, Thielke R, Payne J, Gonzalez N, Conde JG. Research electronic data capture (REDCap)-a metadata-driven methodology and workflow process for providing translational research informatics support. $J$ Biomed Inform. (2009) 42:377-81. doi: 10.1016/j.jbi.2008.08.010

8. Boarder MR, Weisman GA, Turner JT, Wilkinson GF. G protein-coupled P2 purinoceptors: from molecular biology to functional responses. Trends Pharmacol Sci. (1995) 16:133-9. doi: 10.1016/S0165-6147(00)89001-X

9. Bomberg H, Bierbach B, Flache S, Novak M, Bandner-Risch D, Menger $\mathrm{MD}$, et al. Vasopressin aggravates cardiopulmonary bypass-induced gastric mucosal ischemia. Eur Surg Res. (2015) 54:75-86. doi: 10.1159/000368355

10. Gutkowska J, Jankowski M, Lambert C, Mukaddam-Daher S, Zingg HH, McCann SM. Oxytocin releases atrial natriuretic peptide by combining with oxytocin receptors in the heart. Proc Natl Acad Sci USA. (1997) 94:11704-9. doi: 10.1073/pnas.94.21.11704

11. Iliopoulos I, Flores S, Pratap JN, Cooper DS, Cassedy A, Nelson DP. Argininevasopressin therapy in hypotensive neonates and infants after cardiac surgery: response is unrelated to baseline ventricular function. Cardiol Young. (2017) 27:1031-40. doi: 10.1017/S104795111600189X

12. Maybauer MO, Maybauer DM, Enkhbaatar P, Traber DL. Physiology of the vasopressin receptors. Best Pract Res Clin Anaesthesiol. (2008) 22:253-63. doi: 10.1016/j.bpa.2008.03.003

13. Mei Q, Liang BT. P2 purinergic receptor activation enhances cardiac contractility in isolated rat and mouse hearts. Am J Physiol Heart Circ Physiol. (2001) 281:H334-41. doi: 10.1152/ajpheart.2001.281.1.H334

14. Schafer JA, Hawk CT. Regulation of $\mathrm{Na}+$ channels in the cortical collecting duct by AVP and mineralocorticoids. Kidney Int. (1992) 41:255-68. doi: 10.1038/ki.1992.37

15. Zingg $\mathrm{HH}$. Vasopressin and oxytocin receptors. Baillieres Clin Endocrinol Metab. (1996) 10:75-96. doi: 10.1016/S0950-351X(96)8 0314-4

16. Zenteno-Savin T, Sada-Ovalle I, Ceballos G, Rubio R. Effects of arginine vasopressin in the heart are mediated by specific intravascular endothelial receptors. Eur J Pharmacol. (2000) 410:15-23. doi: 10.1016/S0014-2999(00)00853-0

17. Lechner E, Hofer A, Mair R, Moosbauer W, Sames-Dolzer E, Tulzer G. Arginine-vasopressin in neonates with vasodilatory shock after cardiopulmonary bypass. Eur J Pediatr. (2007) 166:1221-7. doi: 10.1007/s00431-006-0400-0

18. Rosenzweig EB, Starc TJ, Chen JM, Cullinane S, Timchak DM, Gersony WM, et al. Intravenous arginine-vasopressin in children with vasodilatory shock after cardiac surgery. Circulation. (1999) 100:Ii182-6. doi: 10.1161/01.CIR.100.suppl_2.II-182

19. Alten JA, Borasino S, Toms R, Law MA, Moellinger A, Dabal RJ. Early initiation of arginine vasopressin infusion in neonates after complex cardiac surgery. Pediatr Crit Care Med. (2012) 13:300-4. doi: 10.1097/PCC.0b013e31822f1753

20. Morrison WE, Simone S, Conway D, Tumulty J, Johnson C, Cardarelli M. Levels of vasopressin in children undergoing cardiopulmonary bypass. Cardiol Young. (2008) 18:135-40. doi: 10.1017/S10479511080 01881

21. Singh VK, Sharma R, Agrawal A, Varma A. Vasopressin in the pediatric cardiac intensive care unit: myth or reality. Ann Pediatr Cardiol. (2009) 2:65-73. doi: 10.4103/0974-2069.52814

22. Kunkes JH, Baker WL, Hammond JA, Gluck J. Vasopressin therapy in cardiac surgery. J Card Surg. (2019) 34:20-7. doi: 10.1111/jocs.13968

23. Ashraf SS, Tian Y, Zacharrias S, Cowan D, Martin P, Watterson K. Effects of cardiopulmonary bypass on neonatal and paediatric inflammatory profiles. Eur J Cardiothorac Surg. (1997) 12:862-8. doi: 10.1016/S1010-7940(97)00261-3

24. Omar S, Zedan A, Nugent K. Cardiac vasoplegia syndrome: pathophysiology, risk factors and treatment. Am J Med Sci. (2015) 349:80-8. doi: 10.1097/MAJ.0000000000000341

25. van Vessem ME, Palmen M, Couperus LE, Mertens B, Berendsen RR, Tops LF, et al. Incidence and predictors of vasoplegia after heart failure surgery. Eur J Cardiothorac Surg. (2017) 51:532-8. doi: 10.1093/ejcts/ezw316

26. Levin MA, Lin HM, Castillo JG, Adams DH, Reich DL, Fischer GW. Early on-cardiopulmonary bypass hypotension and other factors associated with vasoplegic syndrome. Circulation. (2009) 120:1664-71. doi: 10.1161/CIRCULATIONAHA.108.814533

27. Davalos MC, Barrett R, Seshadri S, Walters HL III, Delius RE, Zidan $\mathrm{M}$, et al. Hyponatremia during arginine vasopressin therapy in children following cardiac surgery. Pediatr Crit Care Med. (2013) 14:290-7. doi: 10.1097/PCC.0b013e3182720473

Author Disclaimer: The contents of the project are the authors' sole responsibility and do not necessarily represent official NIH views.

Conflict of Interest: The authors declare that the research was conducted in the absence of any commercial or financial relationships that could be construed as a potential conflict of interest.

Publisher's Note: All claims expressed in this article are solely those of the authors and do not necessarily represent those of their affiliated organizations, or those of the publisher, the editors and the reviewers. Any product that may be evaluated in this article, or claim that may be made by its manufacturer, is not guaranteed or endorsed by the publisher.

Copyright (c) 2021 Raghavan, da Cruz, Kaufman and Osorio Lujan. This is an open-access article distributed under the terms of the Creative Commons Attribution License (CC BY). The use, distribution or reproduction in other forums is permitted, provided the original author(s) and the copyright owner(s) are credited and that the original publication in this journal is cited, in accordance with accepted academic practice. No use, distribution or reproduction is permitted which does not comply with these terms. 